\title{
TOPOGRAPHIC ANALYSIS OF CHANG'E-4 LANDING SITE USING ORBITAL, DESCENT AND GROUND DATA
}

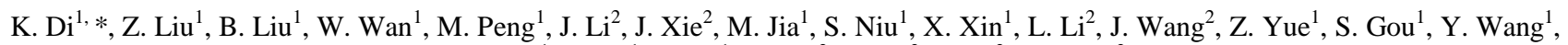

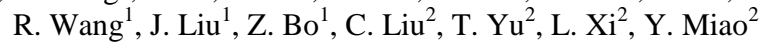 \\ ${ }^{1}$ State Key Laboratory of Remote Sensing Science, Institute of Remote Sensing and Digital Earth, \\ Chinese Academy of Sciences, Beijing, China - (dikc, liuzq, liubin, wanwh, pengman, jiamn, xinxin, yuezy, gousheng, wangyx716, \\ wangrz, liujia2017, bozheng)@ radi.ac.cn; niuvictory@163.com (S. Niu) \\ ${ }^{2}$ Beijing Aerospace Control Center, Beijing, China - 13522132788@139.com (J. Li), 13661277299@139.com (J. Xie), \\ lichunmail@163.com (L.Li), 15210106156@139.com(J. Wang), ckliu2005@126.com (C. Liu), i_am_yty@sina.com (T. Yu), \\ 13521615421@163.com (L.Xi), miaoyi_2008@126.com(Y.Miao)
}

Commission III, ICWG III/II

KEY WORDS: Chang'e-4 Landing Site, Topographic Analysis, Orbital Imagery, Descent Imagery, Ground Imagery

\begin{abstract}
:
The Chang'e-4 (CE-4) probe, which includes a lander and a rover, was successfully landed in Von Kármán crater inside the South Pole-Aitken basin on January 3, 2019. Landing site mapping and topographic analyses have been performed at different scales using orbital, descent and ground data to support mission operations and various scientific investigations. We produced a $0.9 \mathrm{~m}$-resolution seamless digital orthophoto map (DOM) mosaic of the CE-4 landing site region covering $51 \mathrm{~km} \times 30 \mathrm{~km}$ using $100 \mathrm{Lunar}$ Reconnaissance Orbiter Camera (LROC) Narrow-Angle Camera (NAC) images. Within an area of $3.2 \mathrm{~km} \times 3.0 \mathrm{~km}$ of the the DOM around the lander, more than 11,000 craters with diameter greater than $5 \mathrm{~m}$ have been identified and measured. A $0.03 \mathrm{~m}$ - resolution DOM, which covers an area of $211 \mathrm{~m} \times 187 \mathrm{~m}$, was generated using descent images, and has been used as one of the base maps for overall rover traverse planning. During surface operations, local DEMs with $0.02 \mathrm{~m}$ resolution are routinely produced and topographic analysis have been performed at each waypoint using Navcam images and at some locations using Pancam images, to support waypoint-to-waypoint path planning, science target selection and scientific investigations.
\end{abstract}

\section{INTRODUCTION}

The Chang'e-4 (CE-4) probe, which includes a lander and a rover, was launched on December 8, 2018, and successfully landed in Von Kármán crater inside the South Pole-Aitken (SPA) basin on January 3, 2019, marking the first soft landing of human spacecraft on the far side of the moon. The rover, Yutu-2 (Jade Rabbit-2), was released from the lander and started exploration of the lunar surface on the same day. Figure 1 shows the photos of the lander and the rover taken by each other on January 11, 2019. As of March 13, the rover has accomplished the tasks for 3 lunar days and the accumulated travel distance was $163 \mathrm{~m}$ from odometer reading (CLEP, 2019).
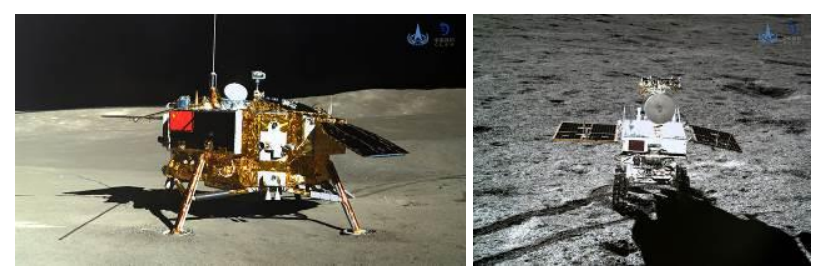

Figure 1. Photos of the CE-4 lander and rover taken by each other on January 11, 2019.

SPA is the largest and oldest impact basin on the moon; it has a diameter of roughly $2,500 \mathrm{~km}$ and depth of $13 \mathrm{~km}$. It could have excavated the oldest, deepest materials from the lower crust and upper mantle. Von Kármán crater is in the northwest of SPA and has a diameter of $180 \mathrm{~km}$. In-situ investigations of Von Kármán crater by CE-4 rover using its cameras, infrared imaging spectrometer, and lunar penetrating radar, are expect to provide insights into SPA basin composition and stratigraphy, the nature of large impact basins and their formation and modification processes, etc (Jia et al., 2018; Huang et al., 2018).

Topographic analysis of CE-4 landing site is important to support mission operations and various scientific investigations. Landing site mapping and topographic analyses have been performed at different scales using orbital, descent and ground data. This paper presents some of the mapping and topographic analysis results achieved by the Planetary Remote Sensing team of State Key Laboratory of Remote Sensing Science, Institute of Remote Sensing and Digital Earth, Chinese Academy of Sciences, in collaboration with the CE-4 teleoperation team of Beijing Aerospace Control Center.

\section{TOPOGRAPHIC CHARACTERIZATION OF VON KÁRMÁN CRATER USING ORBITAL DATA}

\subsection{General Topographic Analysis of Von Kármán Crater Using Orbital Imagery and DEM}

Before the launch of CE-4, orbital mapping products, including Chang'e-2 digital elevation model (DEM) and digital orthophoto map (DOM) (Li et al., 2018), Lunar Reconnaissance Orbiter Camera (LROC) Wide-Angle Camera (WAC) mosaic

\footnotetext{
* Corresponding author
} 
(ASU, 2011, Wagner et al., 2015), and SLDEM2015, a combined product of LOLA laser altimetry and DEMs generated from the Japanese Selenological and Engineering Explorer terrain camera images (Barker et al., 2016), were combinedly used for general analysis of the topographic characteristics of Von Kármán crater and surrounding area.

Figure 2 shows the imagery and topography of Von Kármán crater and surrounding area. From the cross-cutting relations of the large craters in this area, it is easy to discern that Von Kármán crater was formed after Von Kármán $M$, craters Leibnitz, Finsen, Alder, Von Kármán L and Von Kármán L' were all formed after Von Kármán crater. The pre-selected landing region (white box in bottom image of Figure 2) is in the southeast of Von Kármán crater. Many secondary craters and ejecta materials within the landing site region may be traced back to these craters formed after Von Kármán. For example, the NE-SW trending linear features were formed by Finsen (Huang et al., 2018).
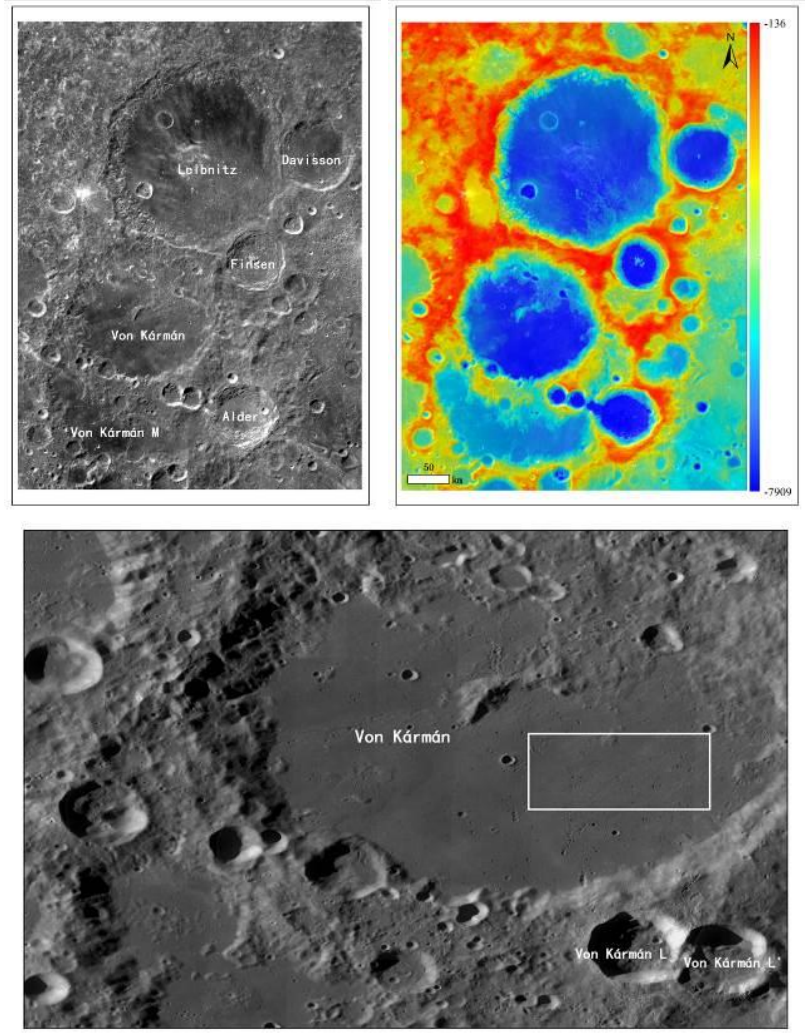

Figure 2. Imagery and topography of Von Kármán crater and surrounding area. Top-left: Chang'e-2 DOM, Top-right: Color coded SLDEM2015, Bottom: LRO WAC mosaic and preselected landing site region (white box) of CE-4.

The landing site region covers the area $\left(176.4^{\circ} \mathrm{E} \sim 178.8^{\circ} \mathrm{E}\right.$, $45.0^{\circ} \mathrm{S} \sim 46.0^{\circ} \mathrm{S}$ ), approximately $51 \mathrm{~km} \times 30 \mathrm{~km}$. Huang et al. (2018) estimated the model age of the mare unit of the region to be $3.6(+0.09,-0.2) \mathrm{Ga}$ based on the size-frequency distribution of craters larger than $2 \mathrm{~km}$ in diameter, indicating the mare unit formed in the Imbrian period.

Figure 3 shows the DEM and slope map of the landing site region in Lambert conformal conic projection with the latitude and longitude of the center of the region being used as the standard parallel and central meridian of the projection. It can be seen in the DEM that the middle and southeast parts of the region are higher than southwest and northeast parts, indicating considerable emplacement of ejecta materials in a NE-SW direction from Finsen crater and possibly from other craters. The resolution of the DEM and slope map is about $42 \mathrm{~m}$ in this region. In this resolution, the landing site region is generally flat with most of the slopes smaller than $15^{\circ}$, larger slopes mostly occur on the walls or rims of craters.
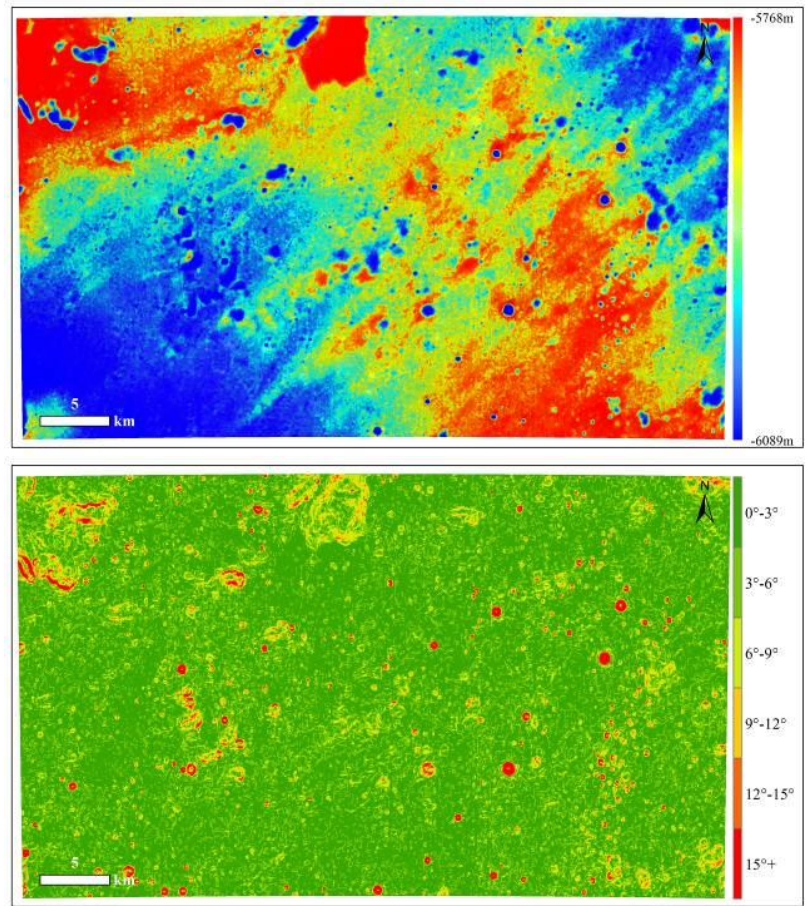

Figure 3. DEM (top) and slope map (bottom) of CE-4 landing site region in Lambert conformal conic projection.

\subsection{High Resolution Mapping of CE-4 Landing Site Region Using LROC NAC images}

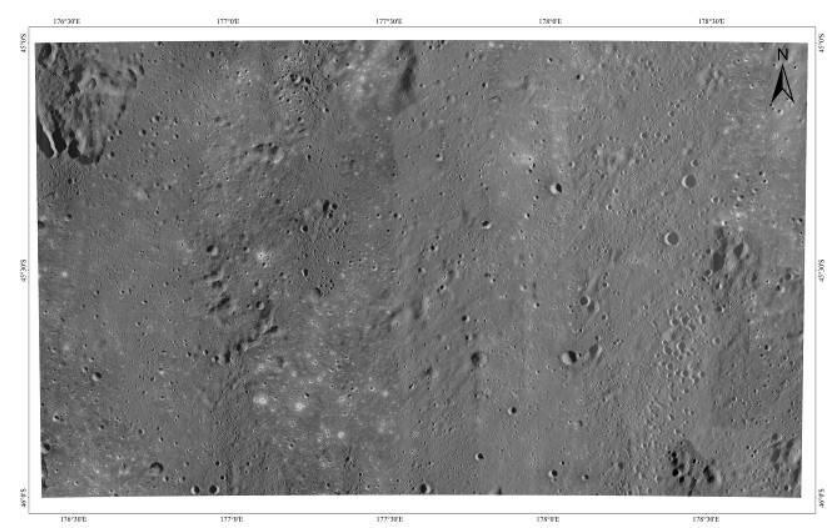

Figure 4. A high-resolution LROC NAC DOM mosaic of CE-4 landing site region in Lambert conformal conic projection.

In order for finer scale topographic analysis, we produced a seamless DOM mosaic of the CE-4 landing site region using 100 LROC Narrow-Angle Camera (NAC) images using the similar techniques for producing a seamless DOM mosaic of Chang'e-5 landing region using LROC NAC images (Di et al., 2018). Figure 4 shows the DOM mosaic in Lambert conformal conic projection. The DOM has the image size of 57,483 columns $\times 34,084$ rows with a ground sample distance of $0.9 \mathrm{~m}$. 
It can be seen that there are many secondary crater clusters overlaid on the mare unit in this region, which can be traced back to several craters in the vicinity, such as Finsen, Von Kármán L, and Von Kármán L'.

\subsection{Statistical Analysis of Craters in CE-4 Landing Site}

Figure 5 shows the high-resolution LROC NAC DOM of the landing site, which covers an area of $3.2 \mathrm{~km} \times 3.0 \mathrm{~km}$. The lander location (see section 3.1 for detail) is marked as a red flag in the map.

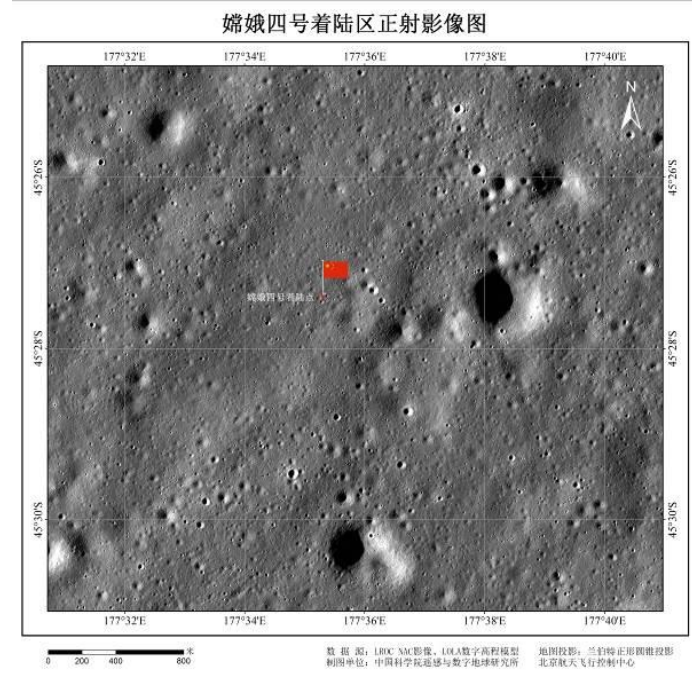

Figure 5. LROC NAC DOM of CE-4 landing site and lander location.

Based on the DOM shown in Figure 5, we identified and measured craters with diameters larger than $3.6 \mathrm{~m}$ (see Figure 6). Overall, there are 11,787 craters identified in this site, among which 11,449 craters are larger than $5 \mathrm{~m}, 4,057$ craters are larger than $10 \mathrm{~m}$ and 28 craters are larger than $100 \mathrm{~m}$. Those 28 craters are shown in light blue color. Figure 7 shows the sizefrequency distribution of craters on a $\log -\log$ plot. The diameter interval is set to be $\sqrt{2} D$ and the crater diameter refers to the middle value of each bin. We found the completeness diameter of the measured craters to be $5 \mathrm{~m}$.

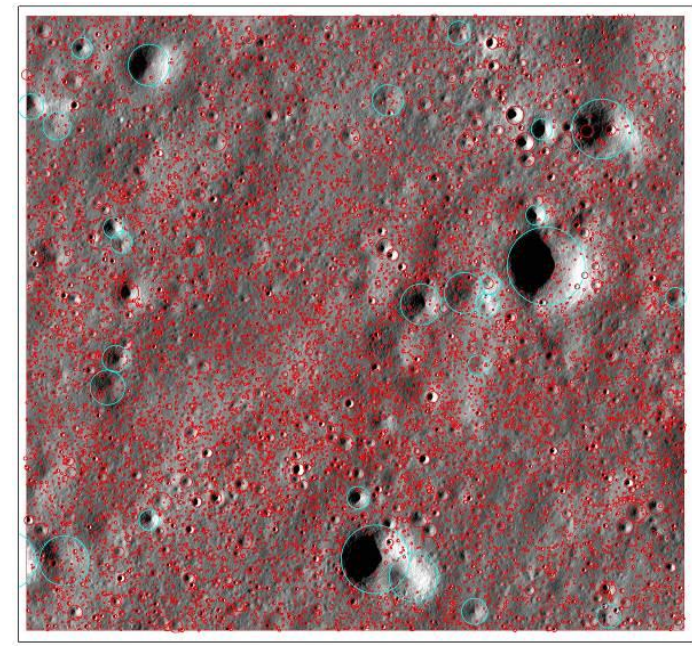

Figure 6. Identified craters in CE-4 landing site.

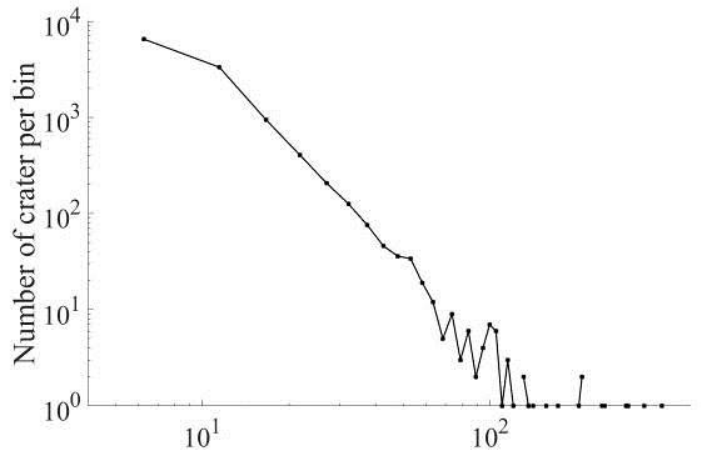

Figure 7. The size-frequency distribution of craters of CE-4 landing site on a log-log plot.

In addition to craters, we also tried to find large rocks in this site. Only 9 rocks with diameters ranging from $3.6 \mathrm{~m}$ to $10.6 \mathrm{~m}$ were identified from inside three larger craters $(>240 \mathrm{~m})$.

\section{DETAILED LANDING SITE MAPPING AND LANDER LOCALIZATION USING DESCENT IMAGES}

\subsection{Lander Localization}

A downward-looking descent camera identical to that on Chang'e- 3 is mounted in the bottom of CE-4. The field of view (FOV) is about $45^{\circ}$ with the image size of $1,024 \times 1,024$ pixels (Liu et al., 2015). The camera took over 4,000 images at 10 frames per second during the descending process. The images have been used for detailed landing site mapping and lander localization.

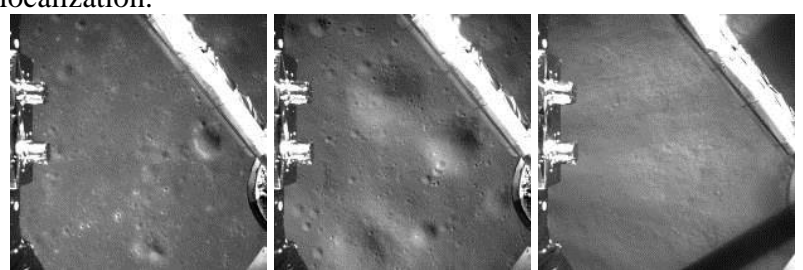

Figure 8. Descent images acquired approximately at $4.2 \mathrm{~km}$,

$100 \mathrm{~m}$ above the lunar surface and after touch down.

Immediately after CE-4 landing, we localized the lander using image feature matching technique based on descent images and orbital base maps such as Chang'e-2 DOM and LROC NAC DOM. The lander location was determined to be $\left(177.588^{\circ} \mathrm{E}\right.$, $45.457^{\circ} \mathrm{S}$ ) (Di et al., 2019). This location is almost the same as directly observed from the LROC NAC image (M1303619844LR) acquired later on 1 February 2019 (ASU, 2019). This verifies that the lander location determined through image feature matching is accurate.

\subsection{Detailed Landing Site Mapping Using Descent Images}

Eighty descent images were selected and used for detailed landing site mapping based on bundle adjustment and image matching techniques (Liu et al., 2015), with a number of control points selected from the LROC NAC DOM of the landing site. As a result, a $0.03 \mathrm{~m}$-resolution DOM of the landing site was produced. Figure 9 shows the DOM that covers an area of 211 $\mathrm{m} \times 187 \mathrm{~m}$. Small features, e.g., meter level craters, can be identified from this very high resolution DOM. The DOM had been used for detailed analysis of the landing site and used as one of the base maps for overall rover traverse planning in the first three lunar days. 


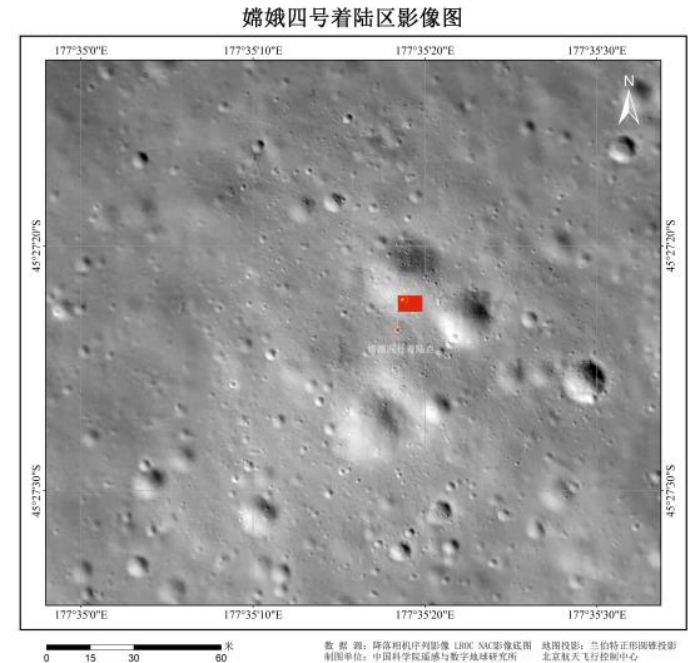

Figure 9. CE-4 landing site DOM generated from descent images.

\section{DETAILED TOPOGRAPHIC MAPPING AND ANALYSIS USING ROVER IMAGES}

Ground data has been acquired by the sensors onboard the rover. Two pairs of stereo cameras, navigation camera (Navcam) and panoramic camera (Pancam), that are mounted on the same camera bar of the rover mast, can obtain 3D terrain information of the lunar surface surrounding the rover. Navcam has an image size of 1,024 pixels $\times 1,024$ pixels, a stereo base of 270 $\mathrm{mm}$ and a FOV of $46.6^{\circ}$ (Liu et al., 2015). Pancam has an image size of 2,352 pixels $\times 1,728$ pixels, a stereo base of 270 $\mathrm{mm}$ and a FOV of $22.9^{\circ} \times 16.9^{\circ}$ (Jia et al., 2018). The rover images have been routinely used for detailed topographic mapping and analysis to support mission operations.

\subsection{Detailed Topographic Mapping and Analysis Using Rover's Pancam Images}

PanCam stereo images are acquired at some locations along the rover traverse for scientific investigation of the site, e.g., surface feature analysis and science target selection. Figure 10 shows a panoramic mosaic of Pancam images taken in the end of the first lunar day of the mission. The rover was about $16 \mathrm{~m}$ (straight-line distance) away from the lander and stopped near the southwest rim of a crater with a diameter of $27 \mathrm{~m}$, which is to the north of the lander. It can be seen that in the vicinity of the lander the terrain is rugged and densely covered with small craters. The rover track is also clearly visible. It should be noted that the bright white stripes in the images are due to light reflections by the rover body.

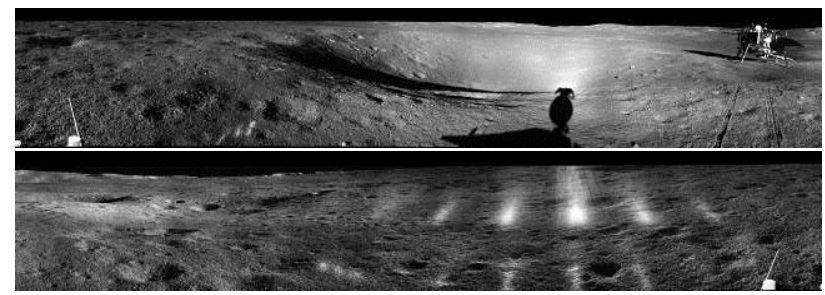

Figure 10. A Pancam image mosaic generated from images taken in the first lunar day.

Unlike Chang'e-3 landing site and many other landing sites where there are many rocks (Di et al, 2016), there are very few rocks on the surface in the landing site, indicating the surface is significantly old. But, we did find several rocks on the wall of the $27 \mathrm{~m}$ crater. As shown in Figure 11, the four relatively "larger" rocks are measured to be 0.2 to $0.3 \mathrm{~m}$ in size.

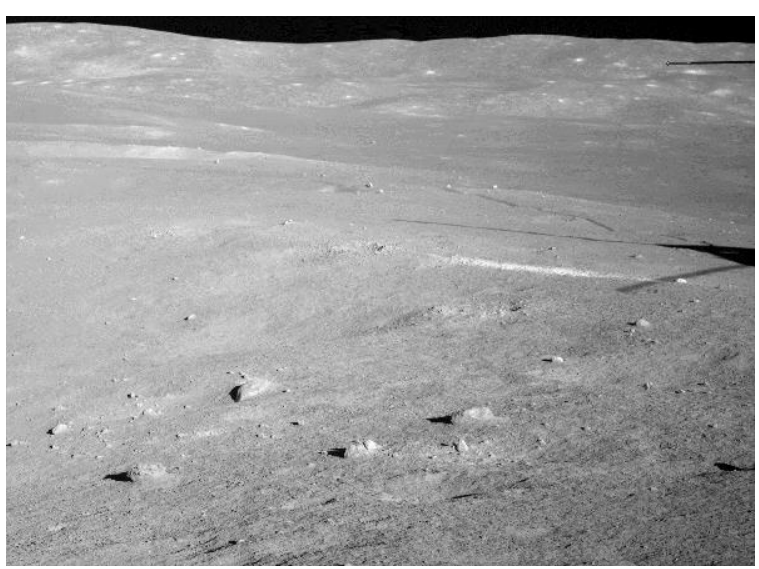

Figure 11. A Pancam image showing several rocks on the wall of the crater to the north of the lander.

Using 58 stereo pairs (two tiers) of Pancam images, we produced a DEM and a DOM that cover an area of $60 \mathrm{~m} \times 60 \mathrm{~m}$ with a resolution of $0.02 \mathrm{~m}$. Figure 12 shows two perspective views of the DOM draped on the DEM. It can be see that the rover traveled on a slope and was very close to the rim of the the $27 \mathrm{~m}$ crater, and there are many other craters along the traverse. Overall, Yutu-2's traveling in this landing site is more challenging than its predecessor Yutu's traveling in Chang'e-3 landing site.

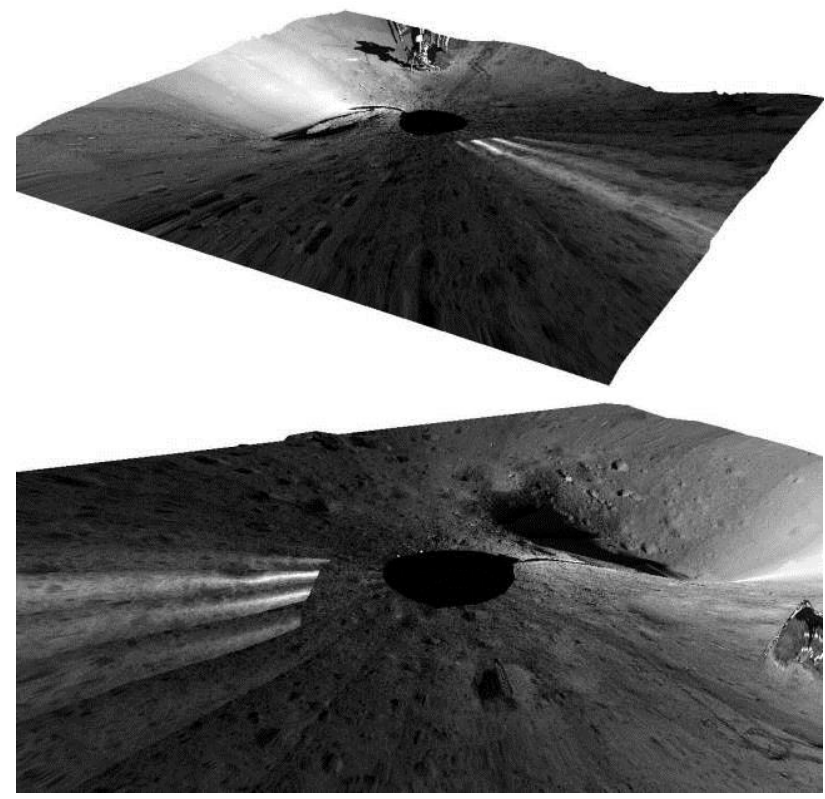

Figure 12. Perspective views of the Pancam DOM draped on the DEM.

\subsection{Detailed Topographic Mapping and Analysis Using} Rover's Navcam Images

In a similar surface operation mode as that in Chang'e-3 mission, Navcam acquires stereo images at every waypoint along the rover traverse. Local DEMs with $0.02 \mathrm{~m}$ resolution were routinely produced at each waypoint using the Navcam images, subsequently detailed topographic analyses, including 
slope computation and hazard identification, were performed to support waypoint-to-waypoint path planning (Peng et al., 2014; Liu et al., 215). Meanwhile, visual localization of the rover was performed using the Navcam stereo images of adjacent waypoints (Wan et al., 2014).

\section{CONCLUSIONS}

This paper presented the mapping and topographic analysis results using orbital, descent and ground data before and during CE-4 mission. We produced a 0.9 m-resolution DOM mosaic of the CE-4 landing site region covering $51 \mathrm{~km} \times 30 \mathrm{~km}$ using 100 LROC NAC images, a $0.03 \mathrm{~m}$-resolution DOM covering $211 \mathrm{~m}$ $\times 187 \mathrm{~m}$ using descent images, 0.02m-resolution DOMs and DEMs along the rover traverse using Pancam and Navcam images. Based on this mapping products, topographic analyses have been performed at different scales and different turnaround times to support mission operations and scientific investigations.

\section{ACKNOWLEDGEMENTS}

This work was supported by the National Natural Science Foundation of China (Grant No. 41671458, 41590851, and 41771488) and the Key Research Program of the Chinese Academy of Sciences (NO. XDPB11). The authors thank all those who worked on the Planetary Data System archive to make the LROC imagery and SLDEM2015 available, Arizona State University for making the LROC WAC mosaic available, China National Space Administration for providing Chang'e-4 descent images and Pancam images, the Lunar and Deep Space Exploration Science Applications Center of the National Astronomical Observatories for providing the Chang'e-2 DOM and DEM.

\section{REFERENCES}

ASU (Arizona State University), 2011. WAC Global Morphologic Map. http://wms.lroc.asu.edu/lroc/view_rdr/WAC_GLOBAL January 2019).

ASU, 2019. Above the Landing Site. http://lroc.sese.asu.edu/posts/1092 (15 February, 2019).

Barker, M.K., Mazarico, E., Neumann, G.A., Zuber, M.T., Haruyama, J., Smith, D.E., 2016. A new lunar digital elevation model from the Lunar Orbiter Laser Altimeter and SELENE Terrain Camera. Icarus, 273, 346-355.

CLEP (China's Lunar and Deep Space Exploration), 2019. The Chang'e-4 lander and rover entered the third lunar night. http://www.clep.org.cn/n5982341/c6805656/content.html (14 March 2019)

Di, K., Xu, B., Peng, M., Yue, Z., Liu, Z., Wan, W., 2016. Rock size-frequency distribution analysis at the Chang'e-3 landing site. Planetary and Space Science, 120, 103-112.

Di, K., Jia, M., Xin, X., Liu, B., Liu, Z., Peng, M., Yue, Z., 2018. High resolution seamless DOM generation over Chang'e5 landing area using LROC NAC images. Int. Arch. Photogramm. Remote Sens. Spatial Inf. Sci., XLII-3, 271-276.
Di, K., Liu, Z., Liu, B. et al., 2019. Chang'e-4 lander localization based on multi-source data. Journal of Remote Sensing, 23(1), 177-184.

Huang, J., Xiao, Z., Flajault, J., Martinot, M., Head, J., Xiao, X., Xie, M., Xiao, L., 2018. Geological Characteristics of Von Kármán Crater, Northwestern South Pole-Aitken Basin: Chang'E-4 Landing Site Region. Journal of Geophysical Research: Planets, 123, 1684-1700

Jia, Y., Zou, Y., Ping, J., Xue, C., Yan, J. and Ning, Y., 2018. The scientific objectives and payloads of Chang'E-4 mission. Planetary and Space Science, 162, 207-215.

Li. C., Liu, J., Ren, X., et al, 2018. Lunar Global High-precision Terrain Reconstruction Based on Chang'e-2 Stereo Images. Geomatics and Information Science of Wuhan University, 43(4), 485-49.

Liu. Z., Di, K., Peng, M., et al., 2015. High precision landing site mapping and rover localization for Chang'e-3 mission. Science China-physics Mechanics \& Astronomy, 58(1), 1-11.

Peng, M., Wan, W., Wu, K., Liu, Z., Li, L., Di, K., Li, L., Miao, Y., Zhan, L., 2014. Topographic mapping capability analysis of Chang'e-3 Navcam stereo images and three-dimensional terrain reconstruction for mission operations. Journal of Remote Sensing, 18(5), 995-1002.

Robinson, M.S., Brylow, S.M., Tschimmel, M,, Humm, D.C., Lawrence, S.J., Thomas, P.C., Denevi, B.W., Bowmancisneros, E., Zerr, J., Ravine, M.A., 2010. Lunar Reconnaissance Orbiter Camera (LROC) Instrument Overview. Space Science Reviews, 150, 81-124.

Wan, W., Liu, Z., Di, K., Wang, B., and Zhou, J., 2014. A Cross-Site Visual Localization Method for Yutu Rover. Int. Arch. Photogramm. Remote Sens. Spatial Inf. Sci., XL-4, 279284.

Wagner, R.V., Speyerer, E.J., Robinson, M.S., \& LROC Team, 2015. New Mosaicked Data Products from the LROC Team. Proceedings of 46th Lunar and Planetary Science Conference, 16-20 March 2015, the Woodlands, Texas, pp. 1473. 